\title{
MELHORIA DA SEGURANÇA DINÂMICA BASEADA EM ANÁLISE ESTOCÁSTICA E METAHEURÍSTICA
}

\author{
Carlos Alberto da Silva Neto* \\ cnetodons.org.br \\ Julio Cesar Stacchini Souza* \\ juliodic.uff.br
}

\author{
Marcus Th. Schilling* \\ schilling@ic.uff.br
}

Marcelos Groetaers dos Santos ${ }^{\dagger}$

marcelseons.org.br

\author{
${ }^{*}$ Universidade Federal Fluminense \\ Rua Passo da Pátria 156, Bloco D, Sala 509 \\ CEP 24210-240, Niterói, RJ \\ ${ }^{\dagger}$ Operador Nacional do Sistema Elétrico (ONS) \\ Rua da Quitanda, 196 \\ CEP 20095-001, Rio de Janeiro, RJ
}

\begin{abstract}
Dynamic Security Improvement Based on Stochastic Analysis and Metaheuristic

This paper presents a new approach for the dynamic security assessment based on the improvement of an index that represents the current probabilistic risk level of the electric grid operation. The index is based on a comprehensive analysis where several electromechanical simulations are performed, considering uncertainties regarding topological configurations, load levels, intertie flows, as well as several different types, locations and duration of faults. These simulations based on detailed models allow for a more accurate performance evaluation of the power system. The optimization process is based on the metaheuristic called Particle Swarm algorithm.
\end{abstract}

KEYWORDS: Metaheuristic, Power system, Stochastic dynamic security, Electrical energy

Artigo submetido em 19/01/2011 (Id.: 1254)

Revisado em 22/03/2011, 10/08/2011

Aceito sob recomendação do Editor Associado Prof. Eduardo N. Asada

\section{RESUMO}

Este artigo apresenta uma nova abordagem para a avaliação da segurança dinâmica de sistemas elétricos de potência baseada na melhoria de um índice que representa o nível de risco operativo probabilístico da rede elétrica. Esse índice é obtido a partir de uma avaliação na qual são realizadas diversas simulações eletromecânicas, considerando as incertezas relacionadas a configurações topológicas, patamares de carga e perfis de intercâmbio, bem como tipos, locais e duração de defeitos. As simulações eletromecânicas com modelos detalhados permitem uma representação mais acurada dos elementos da rede elétrica resultando em um diagnóstico mais confiável. O processo de otimização é baseado na metaheurística denominada algoritmo Enxame de Partículas.

PALAVRAS-CHAVE: Metaheurística, Sistemas de potência, Segurança dinâmica estocástica, Energia elétrica.

\section{INTRODUÇÃO}

Praticamente todos os aspectos da vida moderna como, por exemplo, segurança, saúde, educação e lazer são dependentes do fornecimento adequado da energia elétrica. Esse recurso deve ser disponibilizado de maneira contínua, segura e com qualidade. A operação confiável de sistemas elétri- 
cos de potência busca atender a esses requisitos. A robustez de uma rede elétrica corresponde à sua capacidade de suportar a ocorrência de contingências previstas ou não, sem que haja a sobrecarga de equipamentos ou a violação de limites operativos como as faixas de tensão, por exemplo.

O papel principal dos profissionais envolvidos com a operação de sistemas elétricos é minimizar a ocorrência desses desastres, bem como o impacto dos mesmos, uma vez que é impossível garantir que eles nunca aconteçam. Para isso são realizados estudos utilizando modelos matemáticos que simulam o comportamento do sistema tanto em condições normais quanto de defeito. Essas simulações possibilitam identificar condições operativas inseguras, bem como apontar as ações necessárias para evitar essas situações. Devido à alta complexidade do sistema, que resulta em um número elevado de possíveis configurações de topologia, despachos de geração e níveis de consumo de energia, os especialistas que trabalham nessa área usualmente avaliam deterministicamente os piores cenários possíveis e, a partir deles, definem as regras para a operação mais adequada e segura. A operação de sistemas planejada a partir da "análise do pior caso" resulta em cenários nos quais o sistema tende a sobreviver a distúrbios extremos que, embora raros, poderiam resultar em desligamentos em cascata. Essa abordagem, normalmente é muito conservadora e resulta em soluções não otimizadas do ponto de vista econômico, tendo como exemplo o despacho desnecessário de uma unidade termoelétrica, ou ainda a restrição de intercâmbio energético entre duas áreas. A despeito dessa prática geral, o senso comum diz que é importante ponderar a interpretação dos resultados de acordo com a probabilidade da perturbação e da condição do sistema sob estudo. Essa referência à probabilidade é encontrada dentro dos conjuntos de critérios de estudos, por exemplo, em relatório de pesquisa do Electric Power Research Institute (Anderson et al., 1983) e em documentos do Operador Nacional do Sistema Elétrico (ONS, 2009), que são utilizados na determinação das condições de adequação e segurança de um sistema de potência. Portanto, a necessidade de consideração das incertezas é fartamente reconhecida na literatura (Schilling et al., 2009), a despeito das dificuldades intrinsecamente associadas. Enquanto o cálculo de adequação probabilística considerando análises estáticas encontra-se em razoável estado de maturidade e desenvolvimento, o mesmo não ocorre no que concerne à denominada segurança probabilística considerando análise dinâmica. Isto acontece porque até recentemente, não havia nenhum mecanismo para computar probabilidades nos programas atuais de análise de estabilidade e as ponderações eram realizadas, simplesmente, com base no bom senso ou na experiência. Não obstante, desenvolvimentos recentes mudaram esse panorama (Takahata, 2008; Groetaers dos Santos, 2009).
$\mathrm{Na}$ abordagem adotada neste artigo, a probabilidade do sistema tornar-se instável e as consequências daí decorrentes são examinadas, sendo calculado o grau de exposição do sistema a distúrbios, ou seja, o risco operativo. Esse risco é expresso pelo Índice de Estabilidade Aparente (IEA) (Groetaers dos Santos, 2009), que reflete a expectância de margem negativa de energia. Uma contingência instável resulta em uma margem negativa. Quanto maior for o módulo dessa margem negativa mais severa é a instabilidade, requerendo, por exemplo, um maior corte de carga para o sistema não entrar em colapso. Dessa forma, uma alta expectância da margem negativa, ou seja, um IEA muito negativo, indica que ocorreram muitas contingências instáveis ou contingências muito severas na análise estocástica.

Ao analisar a literatura sobre o problema de otimização da segurança dinâmica de sistemas de potência, é possível fazer uma divisão das diferentes abordagens propostas em dois grandes conjuntos. O primeiro consiste na utilização de algoritmos convencionais determinísticos de otimização, nos quais são inseridas restrições que procuram representar o comportamento dinâmico do sistema elétrico (Kuo and Bose, 1995; Sun et al., 2004; Wang and Yu, 2008; ZarateMinano et al., 2010). O segundo corresponde à utilização de técnicas de inteligência artificial como redes neurais (Karapidakis and Hatziargyriou, 2000; Valette et al., 2008; Voumvoulakis and Hatziargyriou, 2010) ou ainda lógica difusa (Kamwa et al., 2001). Trabalhos mais recentes propõem a utilização combinada dessas metodologias (Assis et al., 2007; Sant'anna et al., 2008), porém ainda sob a perspectiva determinística.

A utilização de metaheurísticas em problemas de otimização em sistemas de potência é bastante diversificada, envolvendo diferentes tópicos tais como despacho econômico (Rudolf and Bayrleithner, 1999) ou máximo carregamento (Chen et al., 2009). Um levantamento extenso dessas aplicações é apresentado em (Lee and El-Sharkawi, 2008). Na área de dinâmica de sistemas de potência é possível encontrar algumas aplicações tais como a identificação dos parâmetros de um modelo dinâmico de carga (Ju et al., 1996) ou o ajuste dos pesos de uma rede neural para a avaliação de segurança dinâmica (Voumvoulakis and Hatziargyriou, 2010). Entretanto, a grande maioria das publicações está relacionada ao projeto ou ajuste de parâmetros de controladores (Mohammadi et al., 2009; Abido, 2001). Um levantamento dessas aplicações é apresentado em (Panigrahi et al., 2010).

Este artigo propõe então uma metodologia flexível para a melhoria da segurança dinâmica, que possibilita a representação detalhada do comportamento dinâmico do sistema elétrico, incluindo um variado espectro de incertezas que se refletem na magnitude do índice IEA, utilizado como função objetivo. No exemplo aqui tratado, o índice de aptidão de cada solu- 
ção candidata corresponde ao IEA associado a cada cenário operativo analisado. O cenário é caracterizado pelo despacho de potência ativa nos geradores. A metaheurística utilizada é baseada em um algoritmo Enxame de Partículas. A metodologia é computacionalmente intensiva, porém possível com os recursos computacionais já disponíveis.

\section{OTIMIZAÇÃO BASEADA EM METAHEU- RÍSTICAS}

O termo metaheurística resulta da combinação da palavra grega heurística que significa descobrir e que está associada a qualquer processo ou método que resulta na solução de problemas, com o prefixo meta que significa além. Essa expressão normalmente é utilizada para definir um processo de busca de alto nível para a descoberta de soluções (Silva Neto and Becceneri, 2009).

Metaheurística é, então, um processo de busca que tenta explorar o espaço das soluções viáveis baseado em alguma estratégia e que incorpora mecanismos para evitar o confinamento em mínimos ou máximos locais. As metaheurísticas apresentam como vantagem a grande capacidade de generalização, uma vez que não há a necessidade de impor condições à função objetivo ou às restrições tais como serem contínuas e diferenciáveis. O processo de busca precisa apresentar alguma aleatoriedade, de forma a permitir uma exploração abrangente do espaço de soluções, mas ao mesmo tempo, precisa ser guiado com algum propósito, normalmente, o de reforçar o bom desempenho das soluções geradas. $\mathrm{O}$ grande desafio está na identificação do conjunto de variáveis de controle e na definição da função aptidão que faça o mapeamento adequado do desempenho ou qualidade da solução gerada. Outro grande desafio dessa metodologia é exatamente o equilíbrio entre os processos de diversificação, ou exploration, e de intensificação, ou exploitation. O primeiro consiste na exploração mais ampla do espaço de soluções, enquanto que o segundo caracteriza a análise mais focada ou intensa.

Algumas tentativas de classificação de metaheurísticas diferenciam aquelas inspiradas na natureza como, por exemplo, os algoritmos genéticos ou evolutivos (Sarimveis and Nikolakopoulos, 2005) e o algoritmo Enxame de Partículas (Kennedy and Eberhart, 1995), daqueles não inspirados na natureza, como por exemplo, o algoritmo GRASP - Greedy Randomized Adaptive Search Procedure (Feo and Resende, 1995) ou o algoritmo da Busca Tabu (Glover, 1989; Glover, 1990). É possível caracterizar ainda aqueles métodos baseados em populações de soluções (e.g. algoritmo genético) ou no aperfeiçoamento de uma solução inicial (e.g. Busca Tabu).

\subsection{Algoritmo Enxame de Partículas}

Esse algoritmo é do tipo evolutivo e seu desenvolvimento foi inspirado na simulação de um sistema social simplificado (Kennedy and Eberhart, 1995). Ele procura reproduzir o deslocamento de um conjunto de indivíduos (bando de pássaros, cardume de peixes ou enxame de insetos) que pode ser caracterizado por um comportamento individualmente aleatório, mas globalmente direcionado. A metodologia, portanto, também é baseada na manipulação de uma população de soluções que é inicializada de forma aleatória. O processo de otimização consiste na busca da posição com maior aptidão, sendo que o espaço de busca corresponde ao conjunto de possíveis posições de todas as partículas. O deslocamento de cada uma delas é afetado pela sua própria trajetória, bem como pelo das partículas que apresentam melhor desempenho. A metodologia tende a preservar as posições com melhor aptidão e descartar as outras. Para tanto, o movimento de cada partícula é afetado tanto pela informação sobre sua melhor posição quanto pelo registro da melhor posição obtida considerando todas as outras partículas. A posição e a velocidade iniciais de cada partícula são definidas aleatoriamente. A cada nova iteração esses valores são atualizados de acordo com as equações (1) e (2). Esse processo é repetido até ser atingido o número máximo de iterações.

$$
v_{i}^{t+1}=w_{i}^{t} v_{i}^{t}+C_{1} f_{R}\left(\text { pbest }_{i}-p_{i}^{t}\right)+C_{2} f_{R}\left(\text { gbest }-p_{i}^{t}\right)
$$

$$
p_{i}^{t+1}=p_{i}^{t}+v_{i}^{t+1}
$$

No cálculo da velocidade de cada partícula, representado pela equação (1), $v_{i}^{t+1}$ é a nova velocidade para a partícula $i$, $w_{i}$ é a inércia da partícula $i, v_{i}^{t}$ é a velocidade anterior da partícula $i, C_{1}$ é o fator de ponderação para o termo pbest ${ }_{i}, f_{R}$ é uma função de geração de números aleatórios no intervalo $[0,1]$, pbest $_{i}$ é a posição com a melhor aptidão obtida pela partícula $i$ até a iteração $t, C_{2}$ é o fator de ponderação para o termo gbest e gbest é a posição melhor aptidão obtida por todo enxame até a iteração $t$.

No cálculo da posição de cada partícula, representado pela equação (2), $p_{i}^{t+1}$ é a nova posição da partícula $i$ e $p_{i}^{t}$ é a posição anterior da partícula $i$.

O processo de exploração do espaço de soluções é governado pelo cálculo da velocidade das partículas e cada termo da equação (1) pode ser interpretado da seguinte forma:

- O primeiro termo da soma corresponde à contribuição da inércia de cada partícula, que controla o impacto da 
variação da velocidade. O fator de inércia $w_{i}$ é reduzido progressivamente de forma a permitir que, inicialmente, a busca tenha uma característica exploratória mais abrangente, mas que posteriormente ela passe a ser mais refinada ou localizada.

- O segundo termo representa o aspecto cognitivo de cada partícula, no qual o conhecimento é baseado na sua própria experiência ou trajetória.

- O terceiro termo representa a influência social, ou seja, a interação entre as partículas.

- Os termos $C_{1}$ e $C_{2}$ representam, respectivamente, os pesos para as contribuições cognitiva e social, que também são ponderados por fatores aleatórios gerados a partir de uma função $f_{R}$ com distribuição uniforme em [0,1].

- O termo pbest $_{i}$ representa a posição da partícula $i$ com a melhor aptidão.

- O termo gbest representa a posição com a melhor aptidão já obtida considerando todas as partículas.

Deve-se ressaltar ainda que tanto a velocidade quanto a posição de cada partícula são vetores definidos no espaço multidimensional correspondente às variáveis de controle do problema, ou seja, $v \in \mathbb{R}^{k}$ e $p \in \mathbb{R}^{k}$, onde $k$ é o número de variáveis de controle. O módulo da velocidade de cada partícula é limitada de acordo com a seguinte expressão:

$$
v_{i}^{M A X}=\frac{p_{i}^{M A X}-p_{i}^{M I N}}{N}, i \in \Omega_{v c}
$$

onde $v_{i}^{M A X}$ é o valor máximo do módulo da componente $i$ da velocidade da partícula, $p_{i}^{M A X}$ e $p_{i}^{M I N}$ são respectivamente os valores máximo e mínimo da coordenada $i$ da partícula e $\Omega_{v c}$ é o conjunto variáveis de controle com dimensão igual a k. O denominador $N$ é um número definido arbitrariamente e que pode afetar o desempenho do algoritmo de tal forma que valores pequenos favorecem uma busca exploratória mais abrangente, enquanto que valores maiores reforçam explorações locais. Neste trabalho foi adotado o valor de $N$ igual a 10 .

Normalmente as coordenadas que caracterizam a posição de uma partícula são facilmente associadas a aspectos concretos do problema de otimização, uma vez que elas refletem os valores que as variáveis de controle podem assumir. Entretanto, é comum que essa associação direta não possa ser feita com relação à velocidade das partículas. Ela corresponde à variação das posições das partículas, o que, em suma, representa o processo de exploração do espaço de soluções. Os valores das velocidades podem ser positivos ou negativos indicando apenas a possibilidade das coordenadas das posições aumentarem ou diminuirem. O cálculo da velocidade máxima apresentado na equação (3) tem o propósito de manter o processo de exploração dentro de condições realistas, uma vez que velocidades muito elevadas poderiam resultar em variações exageradas das coordenadas, podendo fazer com que a partícula fique congelada no limite de alguma coordenada. Mesmo adotando uma estratégia de trocar o sinal da velocidade caso uma coordenada da partícula fique presa no limite, uma velocidade elevada poderia fazer com que a partícula ficasse ricocheteando entre os limites superior e inferior dessa coordenada.

O fluxograma para o algoritmo Enxame de Partículas é apresentado na Figura 1.

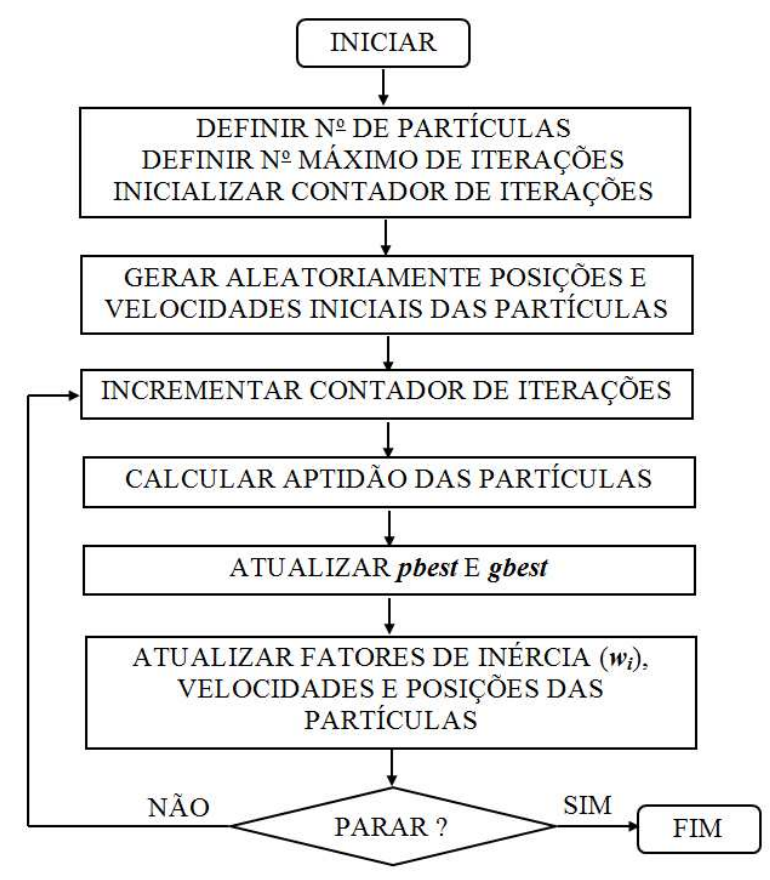

Figura 1: Fluxograma para o Algoritmo Enxame de Partículas.

\section{3 ÍNDICES ESTOCÁSTICOS DE ESTABI- LIDADE}

Dentre os diversos indicadores probabilísticos de estabilidade propostos em (Groetaers dos Santos, 2009) é possível destacar os seguintes: Probabilidade de instabilidade (POI). e o Índice de estabilidade aparente (IEA). 


\subsection{POI - Probabilidade de Instabilidade}

O índice de estabilidade aparente é obtido a partir de uma avaliação estocástica da segurança dinâmica e representa o nível de risco operativo de um sistema elétrico de potência. Nessa avaliação são realizadas diversas simulações eletromecânicas considerando uma variada gama de incertezas.

\subsection{IEA - Índice de Estabilidade Aparente}

O índice de estabilidade aparente é obtido a partir de uma avaliação estocástica da segurança dinâmica e representa o nível de risco operativo de um sistema elétrico de potência. Nessa avaliação são realizadas diversas simulações eletromecânicas considerando uma variada gama de incertezas.

O valor do IEA é obtido a partir de uma análise de contingências na rede elétrica, baseada em simulações eletromecânicas dos defeitos. A margem de energia negativa obtida em cada simulação instável é ponderada pela probabilidade de ocorrência daquela contingência. $\mathrm{O}$ IEA corresponde à combinação linear de todos esses valores ponderados. A margem de energia negativa indica a quantidade de energia que deve ser transferida para outras máquinas menos carregadas, ou, em último caso, o montante de corte de carga necessário para que o sistema volte a ficar estável.

O valor do IEA é obtido a partir de uma análise de contingências na rede elétrica, baseada em simulações eletromecânicas dos defeitos. A margem de energia negativa obtida em cada simulação instável é ponderada pela probabilidade de ocorrência daquela contingência. O IEA corresponde à combinação linear de todos esses valores ponderados. A margem de energia negativa indica a quantidade de energia que deve ser transferida para outras máquinas menos carregadas, ou, em último caso, o montante de corte de carga necessário para que o sistema volte a ficar estável.

É relevante aqui ressaltar que as simulações eletromecânicas completas são fundamentadas na teoria clássica disponível na literatura, e.g. (Kundur, 1994). A rede elétrica foi tratada por elementos passivos, tais como linhas de transmissão e transformadores, modelada via matriz de admitâncias nodais. As cargas foram representadas por admitâncias fixas adicionadas à diagonal da matriz de admitâncias. As potências ativa e reativa geradas são representadas por injeções de correntes nodais. Os geradores foram representados por modelos que variam desde o modelo clássico até os modelos de quarta e quinta ordem, ou seja, máquinas de pólos salientes e pólos lisos, respectivamente. Os controles do tipo reguladores de tensão, limitadores de sobre-excitação, estabilizadores de potência e reguladores de velocidade foram representados a partir de uma grande variedade de opções, similares àquelas existem no sistema elétrico brasileiro. Evidencia-se en- tão que a metodologia proposta emprega soluções clássicas determinísticas de fluxos de potência e estabilidade eletromecânica, durante o processo de otimização. A formalização matemática detalhada do aplicativo computacional utilizado, para a representação dinâmica determinística do sistema pode ser vista em (Groetaers dos Santos, 2009).

De uma forma mais ampla, o índice IEA é um número real adimensional, menor ou igual a zero, que representa o grau de segurança dinâmica do sistema, considerando implicitamente as incertezas de topologia do sistema (contingências de linhas e transformadores), incertezas em patamares de carga (carga pesada, média, etc.), incertezas nos modos de intercâmbio entre áreas elétricas e incertezas nos tipos, locais e durações dos defeitos. No que concerne à interpretação do índice IEA, quanto mais próximo de zero, maior será o grau de segurança do sistema. A grande vantagem do uso deste índice como figura de mérito na avaliação da segurança dinâmica, reside no fato do mesmo embutir intrinsecamente um amplo conjunto de incertezas presentes nos sistemas de potência, traduzindo, portanto, o grau de risco probabilístico real do sistema, sob um ponto de vista dinâmico e globalmente sistêmico. Os procedimentos para o cálculo computacional do IEA são detalhados em (Groetaers dos Santos, 2009) e sua formulação é apresentada no Apêndice Apêndice A.

Formalmente, o IEA corresponde à expectância de margem de energia negativa e quanto menor o módulo desse índice, mais seguro será o sistema, pois ocorrerão menos casos instáveis, ou casos instáveis de menor gravidade. Trata-se portanto de uma avaliação quantitativa e qualitativa, com grande poder discriminatório.

\section{METODOLOGIA PROPOSTA}

A formulação do problema de otimização consiste na maximização do valor do IEA, sujeita às restrições físicas dos equipamentos e limitações operativas da rede elétrica. As variáveis de controle do problema correspondem aos despachos de potência ativa nos geradores.

$$
\begin{aligned}
\text { Maximizar } & I E A\left(\overline{P_{g}}, \overline{Q_{g}}, \bar{V}\right) \\
\text { Sujeito a } & P_{g_{i}}^{M I N} \leq P_{g_{i}} \leq P_{g_{i}}^{M A X}, i=1, N G \\
& Q_{g_{i}}^{M I N} \leq Q_{g_{i}} \leq Q_{g_{i}}^{M A X}, i=1, N G \\
& V_{j}^{M I N} \leq V_{j} \leq V_{j}^{M A X}, j=1, N B \\
& M V A_{l} \leq M V A_{l}^{M A X}, l=1, N L
\end{aligned}
$$

onde $N G$ corresponde ao número de geradores, $N B$ corresponde ao número de barras monitoradas e $N L$ corresponde ao número de linhas de transmissão e transformadores moni- 
torados. A função objetivo do problema, definida na equação (4), corresponde à maximização do IEA, que é função dos despachos de geração ativa $\overline{P_{g}}$ das gerações de reativos $\overline{Q_{g}}$ e o perfil de tensões nas barras $\bar{V}$. As restrições (5) e (6) correspondem às limitações físicas dos equipamentos, que devem ser respeitadas, para evitar sobrecarga. As restrições (7) e (8) correspondem a limitações operativas do sistema elétrico já que não se admite a ocorrência de subtensão ou sobretensão nas barras nem a ocorrência de sobrecarga em circuitos.

O processo de otimização inicia-se pela definição do tamanho do conjunto de soluções candidatas, denominadas partículas, do número máximo de iterações e da inicialização do contador do número de iterações. Ainda nesse passo de inicialização, são definidas, de forma aleatória, "coordenadas" que caracterizam a posição inicial de cada partícula. Os valores das coordenadas correspondem ao despacho de potência ativa nos geradores. Além dos vetores com as coordenadas, também são gerados, de forma aleatória, vetores que caracterizam as velocidades iniciais das partículas. Em seguida é calculada a aptidão de cada partícula. Essa aptidão corresponde ao IEA associado ao vetor de despachos de geração definido para a partícula.

O IEA é obtido a partir de simulações eletromecânicas (Kundur, 1994) de defeitos, considerando uma lista de contingências definida previamente. Para cada contingência definida na lista de contingências, são realizadas três simulações eletromecânicas diferentes. A simulação básica consiste na aplicação de um curto-circuito em uma das extremidades de uma linha de transmissão, o qual é removido após algum tempo pela abertura da linha. A primeira simulação é básica e não se considera nenhum efeito do religamento do circuito. Na segunda simulação é avaliado o comportamento do sistema considerando um religamento bem sucedido, ou seja, a linha é religada após algum tempo e o sistema volta a operar normalmente. Na terceira simulação, é avaliado o comportamento do sistema considerando um religamento mal sucedido. Nesse caso, o curto-circuito volta a ser aplicado e removido de forma definitiva algum tempo depois. Todos esses tempos são descritos no item 5. As simulações estáveis não contribuem para o cálculo do IEA, pois esse índice leva em consideração apenas as margens de energia negativa, que só podem ser calculadas em simulações instáveis (Pai, 1989; Pavella et al., 2000; Jardim, 1994). O cálculo do IEA a partir das margens de energia negativas é descrito em detalhes em (Groetaers dos Santos, 2009).

As aptidões (IEA) calculadas em uma iteração afetam o cálculo da velocidade (1) e, consequentemente, as coordenadas da posição (2), que cada partícula irá ocupar na iteração seguinte. Esse processo de atualização das coordenadas das posições das partículas (despachos de geração) e avaliação da aptidão correspondente (IEA) é repetido até atingir o número máximo de iterações.

\section{SIMULAÇÕES E RESULTADOS}

As simulações foram feitas com o sistema teste New England, disponível na literatura (Athay et al., 1979; Pai, 1989), o qual é composto por uma rede com 3 áreas, 39 barras e 10 geradores. O diagrama unifilar desse sistema, com a identificação das três áreas elétricas, é apresentado na Figura 2.

Para ilustrar a metodologia proposta, o problema resolvido foi obter o despacho de potência ativa que resultou numa condição operativa mais segura do ponto de vista dinâmico, ou seja, aquela com IEA máximo. Dessa forma, o algoritmo de otimização utilizará o IEA como valor da função aptidão para cada solução candidata. A solução do problema de otimização é baseada no algoritmo enxame de partículas considerando uma população de 10 indivíduos e 10 iterações.

A simulação básica de cada uma das contingências, consideradas no cálculo do valor do IEA, consiste na aplicação de um curto-circuito trifásico franco, com duração de 80 ms, em uma das extremidades da linha, seguido da abertura da linha. A duração total da simulação é de 5 segundos.

Foram realizadas três simulações para cada contingência. A primeira simulação corresponde à simulação básica, ou seja, não é considerada a possibilidade de religamento da linha com defeito. Na segunda simulação, o religamento tripolar é realizado com sucesso, ou seja, a linha é religada $250 \mathrm{~ms}$ após a eliminação do defeito. Na terceira simulação o religamento tripolar é realizado sem sucesso, ou seja, um novo curto-circuito trifásico franco é aplicado na barra de defeito $250 \mathrm{~ms}$ após a eliminação do defeito e eliminado outra vez após $80 \mathrm{~ms}$. O religamento bem sucedido ajuda no processo de recuperação do sistema após o distúrbio. Por outro lado o religamento mal sucedido agrava a condição de instabilidade. É interessante observar que no contexto da análise de confiabilidade, a modelagem do religamento assume o papel de medida corretiva. Considerando que a lista de contingências contém 34 circuitos, para cada avaliação do IEA são realizadas 102 simulações eletromecânicas. O processo de otimização consiste na avaliação de 10 soluções candidatas em cada uma das 10 iterações propostas. Dessa forma, são realizadas 10200 simulações para a obtenção da solução final. O tempo total de processamento é de aproximadamente 8000 segundos em um processador Intel Xeon $3 \mathrm{GHz}$, com 3.25 GB de memória RAM.

\subsection{Descrição das Simulações}

Foram feitos testes com diferentes premissas para o cálculo do IEA. Essas premissas estão relacionadas à distribuição da 
probabilidade de ocorrência de defeitos, que pode ser uniforme (equiprovável) ou variar de acordo com o comprimento da linha de transmissão (proporcional), uma vez que, naturalmente, há uma maior probabilidade de ocorrência de defeito em uma linha mais longa do que em uma linha mais curta. No exemplo em questão, a título de simplificação não foram modelados outros tipos e incertezas. Intuitivamente é esperado que a diferença na distribuição das probabilidades de defeito nos circuitos resulte em valores diferentes para o IEA.

Além disso, foram considerados os efeitos da tentativa de religamento dos circuitos na segurança dinâmica do sistema elétrico. A estratégia de religamento busca reduzir o impacto de um defeito passageiro na operação do sistema elétrico. Entretanto, na condição de defeito sustentado, o religamento, que certamente é mal sucedido, resulta em uma condição mais severa para a contingência.

Os dados probabilísticos para a simulação das contingências são apresentados na Tabela 1, que contém a lista de linhas de transmissão que serão avaliadas. Para cada linha há a descrição do local de aplicação do curto-circuito, e as probabilidades de defeito para a distribuição equiprovável e a distribuição proporcional. Note que a distribuição equiprovável corresponde a $1 / 34=0,02941$.

Os testes realizados buscaram identificar inicialmente a influência da distribuição da probabilidade de ocorrência de defeitos, que, conforme já mencionado anteriormente, pode ser equiprovável ou proporcional. Em seguida foi avaliado o efeito do religamento, considerando simulações sem religamento, com religamento sempre bem sucedido ou ainda com religamento que pode ser bem ou mal sucedido. A combinação dessas premissas resultou em um conjunto de quatro testes.

No primeiro teste, o IEA é calculado considerando uma distribuição equiprovável das contingências e sem considerar o religamento dos circuitos. Essa avaliação é interessante porque seu resultado pode ser cotejado com uma avaliação clássica determinística. Em essência trata-se de um mero contador normalizado de eventos.

No segundo teste, o IEA é calculado considerando as probabilidades de ocorrência de defeito proporcionais aos comprimentos dos circuitos, mas ainda sem considerar o religamento dos circuitos. Neste caso já evidencia-se de forma irrefutável o efeito das incertezas.

No terceiro teste, o IEA é calculado considerando as probabilidades de ocorrência de defeito proporcionais aos comprimentos dos circuitos e somente o efeito dos religamentos bem sucedidos é considerado. Nessa avaliação introduz-se o efeito da medida corretiva.
Tabela 1: Dados Estocásticos para as Simulações das Contingências.

\begin{tabular}{|c|c|c|c|c|c|}
\hline & Barra & Barra & Barra de & \multicolumn{2}{|c|}{ Probabilidade de Defeito (\%) } \\
\cline { 5 - 6 } \# CTG & de & para & Curto & Equiprovável & Proporcional \\
\hline 1 & 30 & 31 & 30 & 0,02941 & 0,06915 \\
\hline 2 & 30 & 1 & 30 & 0,02941 & 0,05588 \\
\hline 3 & 31 & 32 & 31 & 0,02941 & 0,02543 \\
\hline 4 & 31 & 25 & 31 & 0,02941 & 0,01446 \\
\hline 5 & 32 & 33 & 32 & 0,02941 & 0,02802 \\
\hline 6 & 32 & 18 & 32 & 0,02941 & 0,02176 \\
\hline 7 & 33 & 34 & 33 & 0,02941 & 0,01691 \\
\hline 8 & 33 & 14 & 33 & 0,02941 & 0,01723 \\
\hline 9 & 34 & 35 & 34 & 0,02941 & 0,00433 \\
\hline 10 & 34 & 37 & 34 & 0,02941 & 0,01659 \\
\hline 11 & 35 & 36 & 35 & 0,02941 & 0,01316 \\
\hline 12 & 35 & 11 & 35 & 0,02941 & 0,01377 \\
\hline 13 & 36 & 37 & 36 & 0,02941 & 0,00773 \\
\hline 14 & 37 & 38 & 37 & 0,02941 & 0,04795 \\
\hline 15 & 38 & 1 & 38 & 0,02941 & 0,07068 \\
\hline 16 & 39 & 11 & 39 & 0,02941 & 0,00723 \\
\hline 17 & 39 & 13 & 39 & 0,02941 & 0,00723 \\
\hline 18 & 13 & 14 & 13 & 0,02941 & 0,01702 \\
\hline 19 & 14 & 15 & 14 & 0,02941 & 0,03637 \\
\hline 20 & 15 & 16 & 15 & 0,02941 & 0,01636 \\
\hline 21 & 16 & 17 & 16 & 0,02941 & 0,01410 \\
\hline 22 & 16 & 19 & 16 & 0,02941 & 0,03142 \\
\hline 23 & 16 & 21 & 16 & 0,02941 & 0,02393 \\
\hline 24 & 16 & 24 & 16 & 0,02941 & 0,00817 \\
\hline 25 & 17 & 18 & 17 & 0,02941 & 0,01342 \\
\hline 26 & 17 & 27 & 17 & 0,02941 & 0,03044 \\
\hline 27 & 21 & 22 & 21 & 0,02941 & 0,02445 \\
\hline 28 & 22 & 23 & 22 & 0,02941 & 0,01718 \\
\hline 29 & 23 & 24 & 23 & 0,02941 & 0,04587 \\
\hline 30 & 25 & 26 & 25 & 0,02941 & 0,05253 \\
\hline 31 & 26 & 27 & 26 & 0,02941 & 0,02422 \\
\hline 32 & 26 & 28 & 26 & 0,02941 & 0,07848 \\
\hline 33 & 26 & 29 & 26 & 0,02941 & 0,10349 \\
\hline 34 & 28 & 29 & 28 & 0,02941 & 0,02502 \\
\hline & & & & & \\
\hline
\end{tabular}

No quarto teste, o IEA é calculado considerando as probabilidades de ocorrência de defeito proporcionais aos comprimentos dos circuitos. Para representar o efeito do religa- 


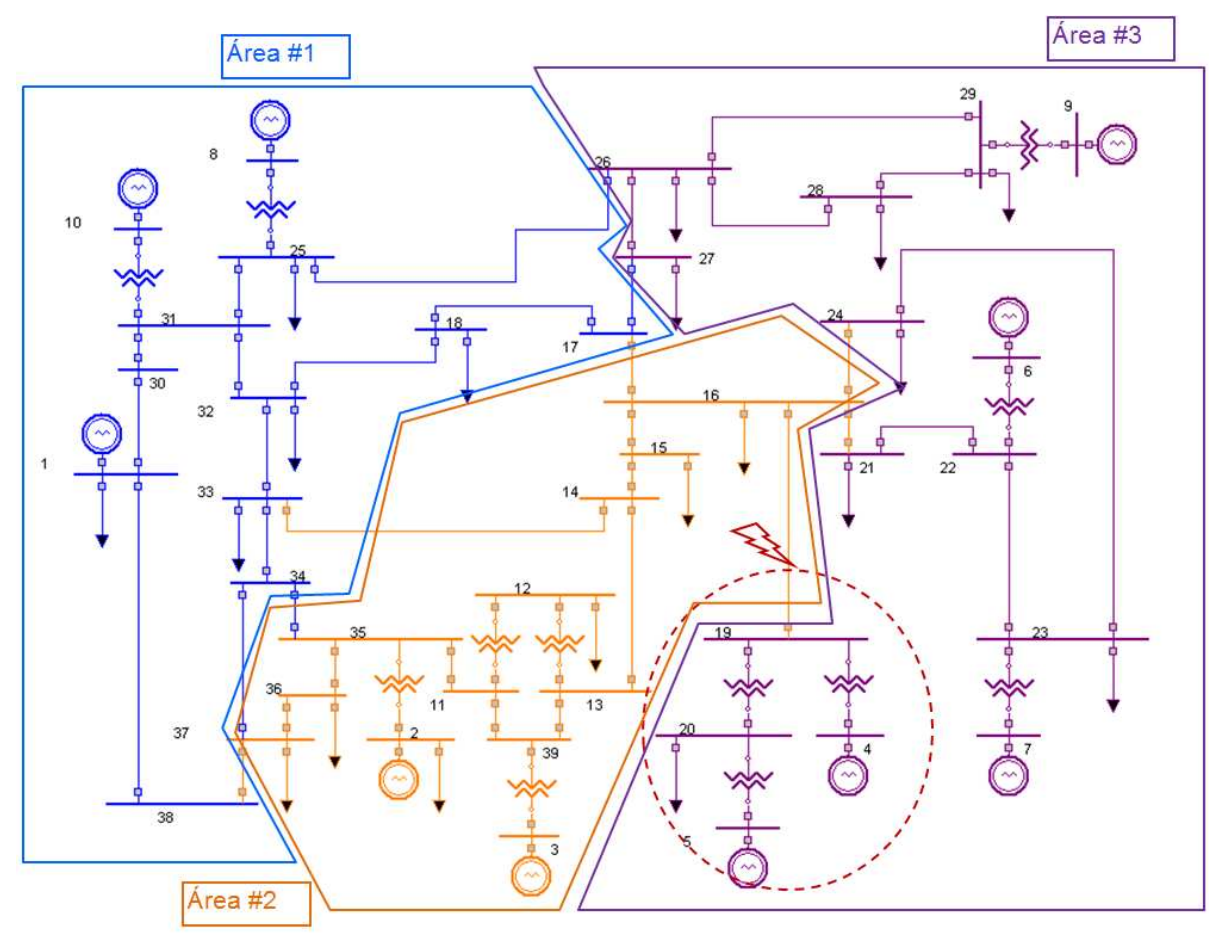

Figura 2: Diagrama Unifilar do Sistema Elétrico de Teste (39 Barras).

mento dos circuitos é adotada uma premissa de que em $80 \%$ dos casos o religamento é bem sucedido, enquanto que em $20 \%$ ele resulta em falha.

A Tabela 2 apresenta uma descrição sumária da premissa de cada teste e os resultados obtidos.

Tabela 2: POI e IEA para os 4 Testes.

\begin{tabular}{|c|c|c|c|c|c|c|}
\hline \multicolumn{3}{|c|}{ Teste } & \multicolumn{2}{c|}{ Caso Base } & \multicolumn{2}{c|}{ Melhor Solução } \\
\hline № & Probabilidade & Religamento & POI & IEA & POI & IEA \\
\hline 1 & Equiprovável & Não & 0,20587 & $-53,80$ & 0,11764 & $-0,33$ \\
\hline 2 & Proporcional & Não & 0,29142 & $-57,83$ & 0,12351 & $-1,54$ \\
\hline 3 & Proporcional & Sempre OK & 0,05644 & $-13,58$ & 0,02445 & $-2,52$ \\
\hline 4 & Proporcional & OK+Falha & 0,16025 & $-21,96$ & 0,61940 & $-8,93$ \\
\hline
\end{tabular}

Como já foi mencionado anteriormente, o índice IEA é um número negativo que quanto mais próximo de zero, melhor é o nível de segurança do sistema elétrico. Na análise dos resultados numéricos para o IEA foi considerado o valor absoluto desse índice de tal forma que quanto menor for esse número melhor será a solução. Ao comparar os resultados do cálculo do IEA, antes do processo de otimização e sem religamento, ou seja, considerando apenas o efeito da distribuição equiprovável de probabilidade de defeitos contra o da distribuição proporcional aos comprimentos das linhas, vide valores destacados na Tabela 2, o módulo do valor calculado para a distribuição equiprovável $(-53,80)$ é ligeiramente inferior ao da distribuição proporcional $(-57,83)$. Esse resultado indica que a distribuição de probabilidades proporcional resultou em uma maior expectância de margem de energia negativa.

A Figura 3 apresenta um gráfico comparando a expectância de margem de energia negativa para esses dois casos. Nesse gráfico, a legenda CBE corresponde aos resultados do caso base com distribuição equiprovável das contingências, enquanto que a legenda CBP, corresponde aos resultados do caso base com distribuição proporcional. No caso com distribuição equiprovável existe uma contingência que resulta em expectância de margem de energia negativa no intervalo entre 0 e $0,5 \mathrm{MW}$, cinco contingências no intervalo entre 0,5 MW e $5 \mathrm{MW}$, nenhuma no intervalo entre $5 \mathrm{MW}$ e $50 \mathrm{MW}$ e uma com margem de energia superior a $50 \mathrm{MW}$. No caso com distribuição proporcional existem duas contingências que resultam em expectância de margem de energia negativa no intervalo entre 0 e $0,5 \mathrm{MW}$, três contingências no intervalo entre $0,5 \mathrm{MW}$ e $5 \mathrm{MW}$, uma no intervalo entre $5 \mathrm{MW}$ e $50 \mathrm{MW}$ e uma com margem de energia superior a 50 MW. Dessa forma é possível confirmar que, apesar dos resultados muito próximos, a diferença no IEA, mencionada anteriormente, pode ser explicada pela contingência que resulta em uma expectância de margem de energia no intervalo entre $5 \mathrm{MW}$ e $50 \mathrm{MW}$. 


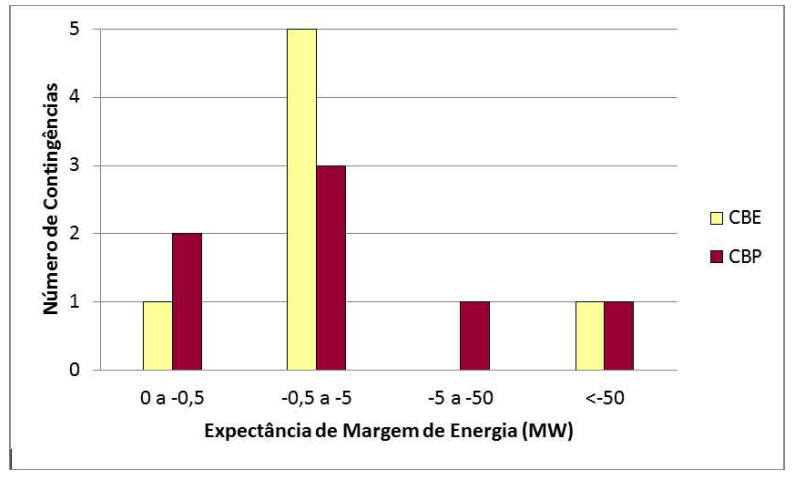

Figura 3: Expectância de Margem de Energia para Distribuição Equiprovável (CBE) e Proporcional (CBP).

Nos quatro testes é possível observar a redução do índice de estabilidade aparente (IEA) obtida pelo processo de otimização. Essa redução pode ser explicada, em parte, pela redução da probabilidade de instabilidade (POI) e em parte pela redução da severidade das contingências. Contingências menos severas resultam em margens de estabilidade negativa menores.

A redução na POI, observada nos três primeiros testes, mostra que houve uma redução no número de simulações instáveis nesses testes. No quarto teste a POI aumentou em relação ao caso base, indicando que houve um aumento do número de casos instáveis. Entretanto, a redução do IEA indica que a severidade da instabilidade desses casos foi menor.

A seguir serão analisados os resultados do processo de otimização para cada teste individualmente.

\subsubsection{Teste \#1 - Contingências equiprováveis, sem religamento}

Nesse caso, o IEA da melhor solução obtida tem valor igual a $-0,33$, bem abaixo do valor do caso base $(-53,80)$, indicando uma melhoria significativa no nível de segurança dinâmica do sistema. A Figura 4 apresenta um gráfico mostrando a redução da expectância de margem de energia negativa proporcionada pelo processo de otimização. Nesse gráfico, a legenda CBE corresponde aos resultados do caso base com distribuição equiprovável das contingências, enquanto que a legenda T1, corresponde aos resultados obtidos no processo de otimização do teste \#1, que não leva em consideração o efeito do religamento. No caso base original existe uma contingência que resulta em expectância de margem de energia negativa no intervalo entre 0 e $0,5 \mathrm{MW}$, cinco contingências no intervalo entre $0,5 \mathrm{MW}$ e $5 \mathrm{MW}$, nenhuma no intervalo entre $5 \mathrm{MW}$ e $50 \mathrm{MW}$ e uma com margem de energia superior a 50 MW. Para a melhor solução obtida existem apenas quatro contingências com expectância de margem negativa no inter- valo entre 0 e 0,5 MW. Esse resultado confirma a redução significativa da probabilidade de contingências instáveis e da expectância de margem de energia negativa para o sistema.

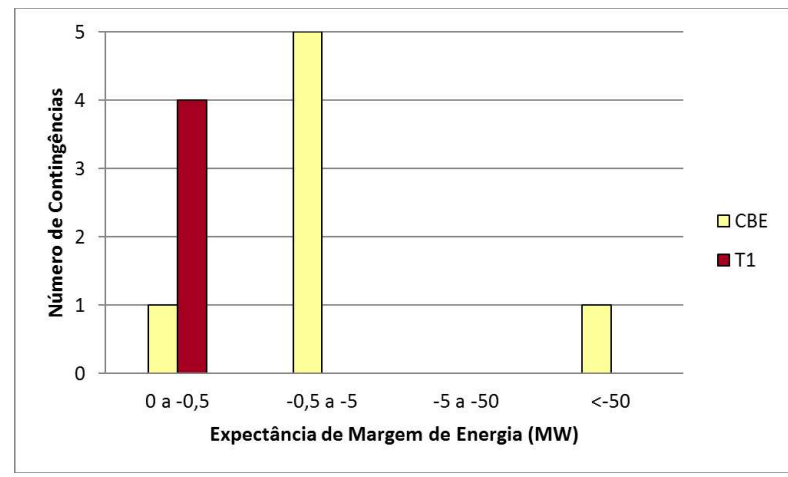

Figura 4: Expectância de Margem de Energia Negativa para Teste \#1 - Caso Original (CBE) e Melhorado (T1).

Um exemplo da redução da severidade das contingências foi observado na perda do circuito 16-19, que resulta na pior margem de energia negativa considerando o despacho original do caso base. Essa contingência causa o ilhamento das barras 4, 519 e 20 da área \#3 (ver destaque na Figura 2). Nesse conjunto de barras ilhadas há um forte desbalanço entre a carga e a geração para o despacho do caso base. A carga da ilha é igual a $628 \mathrm{MW}$ (Barra 20), enquanto que a geração corresponde à soma dos despachos dos geradores 4 e 5, em destaque na Tabela 3. No caso base essa geração é igual a 1140 MW. Para a melhor solução obtida o valor da geração passa a ser igual a $700 \mathrm{MW}$. Essa redução é suficiente para manter o equilíbrio da carga com a geração e, com isso, a contingência deixa de ser crítica, pois o sistema passa a ser estável.

As Figuras 5(a) e 5(b) mostram o comportamento da diferença entre o ângulo dos rotores de geradores localizados nas três áreas do sistema, na simulação dessa contingência.

O gerador G1 está localizado na área \#1, o G2 na área \#2 e o G4 na ilha da área \#3. A Figura 5(a), que corresponde ao caso base (CBE) mostra o gerador G4 perdendo a estabilidade, enquanto que a Figura 5(b), que corresponde à melhor solução obtida (T1), mostra o mesmo gerador recuperando o sincronismo após uma grande excursão angular em relação às outras máquinas do sistema.

\subsubsection{Teste \#2 - Contingências não equiprováveis, sem religamento}

Nesse caso, o IEA da melhor solução obtida tem valor igual a $-1,54$, bem abaixo do valor do caso base $(-57,83)$, indicando uma melhoria significativa no nível de segurança dinâmica do sistema. A Figura 6 apresenta um gráfico mostrando a 
Tabela 3: Comparação dos Despachos de Geração para Distribuição Equiprovável (Teste \#1).

\begin{tabular}{|c|c|c|}
\cline { 2 - 3 } \multicolumn{1}{c|}{} & \multicolumn{2}{c|}{ Ger } \\
\cline { 2 - 3 } \multicolumn{1}{c|}{} & CBE & T1 \\
\hline 1 & 1000 & 1900 \\
\hline 2 & 520 & 443 \\
\hline 3 & 650 & 900 \\
\hline 4 & 632 & 500 \\
\hline 5 & 508 & 200 \\
\hline 6 & 650 & 700 \\
\hline 7 & 560 & 500 \\
\hline 8 & 540 & 100 \\
\hline 9 & 830 & 700 \\
\hline 10 & 250 & 200 \\
\hline Total & 6140 & 6143 \\
\hline
\end{tabular}

redução da expectância de margem de energia negativa proporcionada pelo processo de otimização. Nesse gráfico, a legenda CBP corresponde aos resultados do caso base com distribuição proporcional das contingências, enquanto que a legenda $\mathrm{T} 2$, corresponde aos resultados obtidos no processo de otimização do teste \#2, que não leva em consideração o efeito do religamento. No caso original existem duas contingências que resultam em expectância de margem de energia negativa no intervalo entre 0 e $0,5 \mathrm{MW}$, três contingências no intervalo entre $0,5 \mathrm{MW}$ e $5 \mathrm{MW}$, uma no intervalo entre $5 \mathrm{MW}$ e $50 \mathrm{MW}$ e uma com margem de energia superior a $50 \mathrm{MW}$. No caso otimizado existem cinco contingências com expectância de energia negativa no intervalo entre 0 e 0,5 MW e apenas uma com expectância de margem negativa no intervalo entre 0,5 MW e $5 \mathrm{MW}$. Esse resultado confirma a redução significativa da probabilidade de contingências instáveis e da expectância de margem de energia negativa para o sistema, tal como ocorrido no primeiro teste.

\subsubsection{Teste \#3 - Contingências não equiprováveis, religamento sempre com sucesso}

Nesse caso, o IEA da melhor solução obtida tem valor igual a $-2,52$, também abaixo do valor do caso base $(-13,58)$. A Figura 7 apresenta um gráfico mostrando a redução da expectância de margem de energia negativa proporcionada pelo processo de otimização. Nesse gráfico, a legenda CBP corresponde aos resultados do caso base com distribuição proporcional das contingências, enquanto que a legenda T3, corresponde aos resultados obtidos no processo de otimização do teste \#3, que leva em consideração apenas o efeito do religamento bem sucedido. No caso original não existe ne-

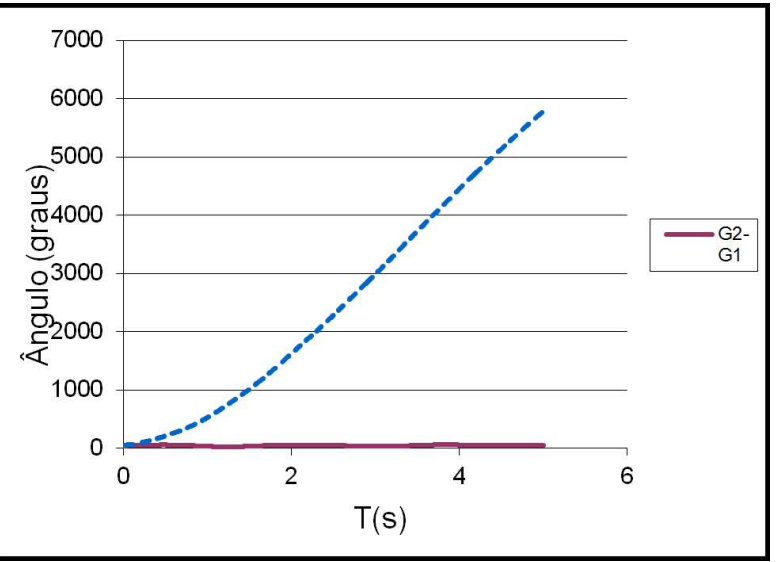

(a)

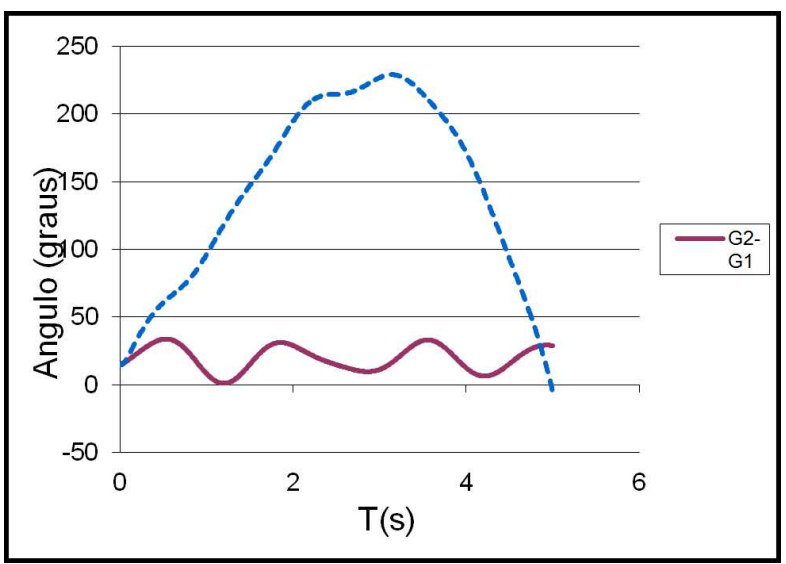

(b)

Figura 5: (a) Diferença do Ângulo dos Rotores para o Despacho Original (CBE). (b) Diferença do Ângulo dos Rotores para o Despacho Otimizado (T1).

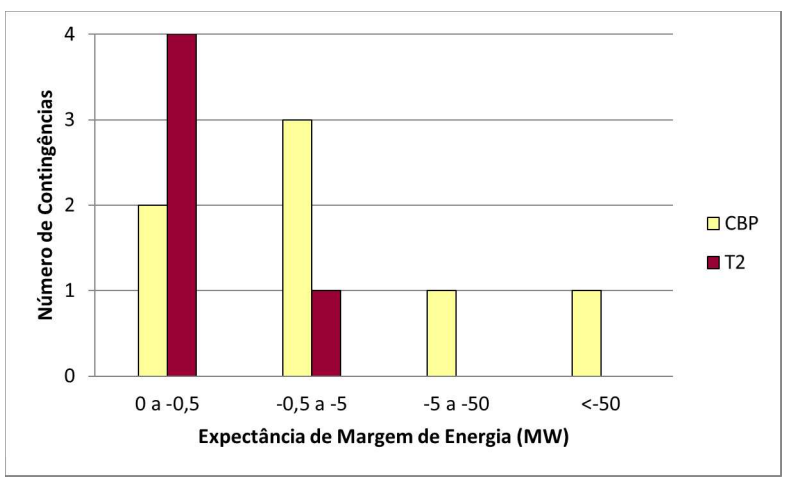

Figura 6: Expectância de Margem de Energia Negativa para Teste \#2 - Caso Original (CBP) e Melhorado (T2).

nhuma contingência que resulte em expectância de margem de energia negativa no intervalo entre 0 e $0,5 \mathrm{MW}$, existe 
uma contingência no intervalo entre $0,5 \mathrm{MW}$ e $5 \mathrm{MW}$, uma no intervalo entre $5 \mathrm{MW}$ e $50 \mathrm{MW}$ e nenhuma com margem de energia superior a $50 \mathrm{MW}$. Para a melhor solução obtida, existe apenas uma contingência que resulte em expectância de margem de energia negativa no intervalo entre 0,5 MW e 5 MW. Mais uma vez é possível observar a redução da probabilidade de contingências instáveis e expectância de margem de energia negativa.

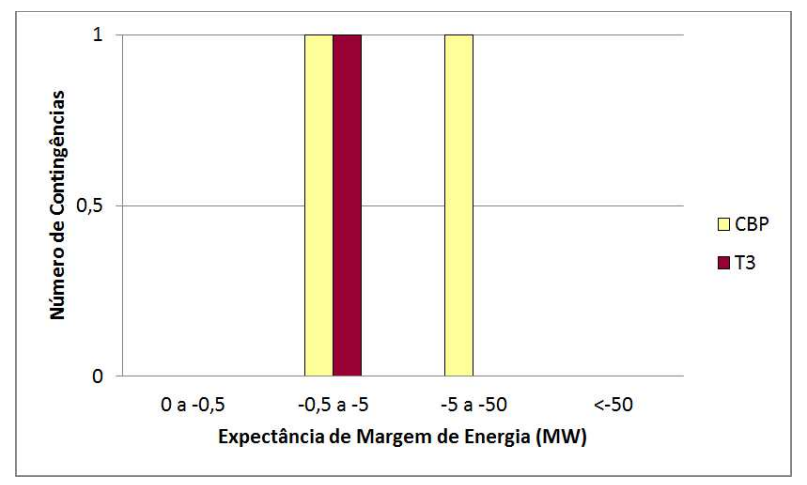

Figura 7: Expectância de Margem de Energia Negativa para Teste\#3 - Caso Original (CBP) e Melhorado (T3).

\subsubsection{Teste \# 4 - Contingências não equiprová- veis, possível falha no religamento}

Nesse caso, o IEA da melhor solução obtida tem valor igual a -8,93, mais uma vez ficando abaixo do valor do caso base $(-21,96)$. Entretanto, é possível observar um aumento no índice de probabilidade de instabilidade (POI), que passou de $(0,16025)$ no caso original para $(0,61940)$ no caso melhorado.

A Figura 8 apresenta um gráfico mostrando a redução da expectância de margem de energia negativa proporcionada pelo processo de otimização. Nesse gráfico, a legenda CBP corresponde aos resultados do caso base com distribuição proporcional das contingências, enquanto que a legenda T4, corresponde aos resultados obtidos no processo de otimização do teste \#4, que leva em consideração o efeito do religamento que tanto pode ser bem sucedido quanto mal sucedido. No caso original existem três contingências que resultam em expectância de margem de energia negativa no intervalo entre 0 e 0,5 MW, catorze contingências no intervalo entre 0,5 MW e $5 \mathrm{MW}$, sete no intervalo entre $5 \mathrm{MW}$ e $50 \mathrm{MW}$ e uma com margem de energia superior a $50 \mathrm{MW}$. Para a melhor solução obtida, existem sessenta e seis contingências que resultam em expectância de margem de energia negativa no intervalo entre 0 e $0,5 \mathrm{MW}$, dezessete contingências no intervalo entre $0,5 \mathrm{MW}$ e $5 \mathrm{MW}$, três no intervalo entre $5 \mathrm{MW}$ e $50 \mathrm{MW}$ e nenhuma com margem de energia superior a $50 \mathrm{MW}$.

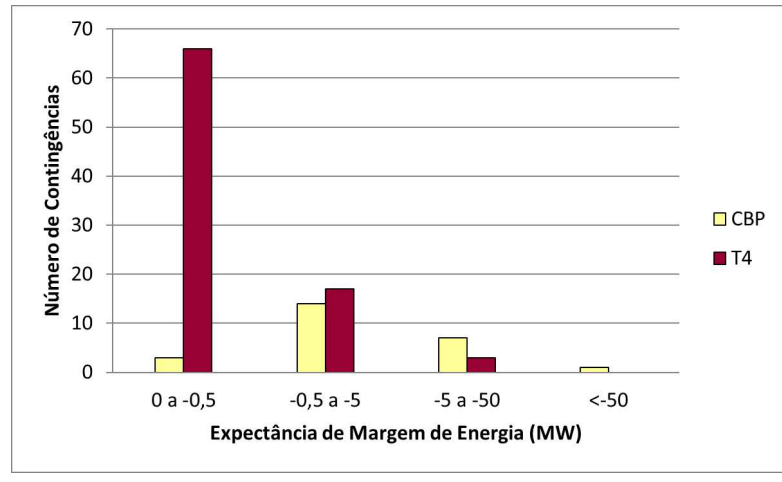

Figura 8: Expectância de Margem de Energia Negativa para Teste \#4 - Caso Original (CBP) e Melhorado (T4).

$\mathrm{O}$ aumento do número de contingências com alguma expectância de margem de energia negativa explica o aumento do POI na última linha da Tabela 2 . O baixo valor da expectância de margem de energia negativa nesse maior número de contingências instáveis indica que a adoção de medidas corretivas de pequena monta já seria suficiente para evitar a instabilidade do sistema, justificando a melhoria no nível de segurança do sistema elétrico. A existência de contingências com expectância de energia negativa na faixa entre $5 \mathrm{MW}$ e $50 \mathrm{MW}$ explica o elevado valor do IEA, quando comparado aos outros testes na Tabela 3. Uma possível razão para esse comportamento é o efeito do religamento com falha, que é considerado nesse teste. A reinserção da falta agrava a condição de instabilidade do sistema, degradando a margem de estabilidade.

O aumento do contador de número de casos instáveis, ou seja, do índice POI, indica que a otimização multiobjetivo baseada na frente de Pareto, tendo como funções objetivo o IEA em um eixo e a POI em outro, poderia auxiliar no processo de escolha da solução com melhor desempenho, considerando o compromisso entre a redução simultânea da expectância de margem de energia negativa e do número de contingências instáveis.

A Tabela 4 apresenta os despachos de geração dos casos base e aqueles correspondentes às melhores soluções em cada teste realizado.

\subsection{Comentários Adicionais}

Deve-se ressaltar que a metodologia para cálculo do IEA permite a avaliação direta de outros tipos de incertezas, tais como, tipos de faltas (ex. monofásica), contingências em transformadores, patamares de carga diversos ponderados por suas respectivas probabilidades de ocorrência e diferentes perfis de intercâmbio entre áreas, também ponderados por suas probabilidades. As incertezas associadas ao parque 
Tabela 4: Comparação dos Despachos de Geração.

\begin{tabular}{|c|c|c|c|c|c|c|}
\cline { 2 - 7 } \multicolumn{1}{c|}{} & \multicolumn{5}{c|}{ Ger } \\
\cline { 2 - 7 } \multicolumn{1}{c|}{} & CBE & T1 & CBP & T2 & T3 & T4 \\
\hline 1 & 1000 & 1900 & 1000 & 1739 & 1300 & 2000 \\
\hline 2 & 520 & 443 & 520 & 125 & 345 & 547 \\
\hline 3 & 650 & 900 & 650 & 800 & 400 & 400 \\
\hline 4 & 632 & 500 & 632 & 0 & 100 & 300 \\
\hline 5 & 508 & 200 & 508 & 600 & 300 & 900 \\
\hline 6 & 650 & 700 & 650 & 400 & 900 & 800 \\
\hline 7 & 560 & 500 & 560 & 300 & 600 & 400 \\
\hline 8 & 540 & 100 & 540 & 1000 & 700 & 400 \\
\hline 9 & 830 & 700 & 830 & 700 & 600 & 300 \\
\hline 10 & 250 & 200 & 250 & 500 & 900 & 1000 \\
\hline Total & 6140 & 6143 & 6140 & 6164 & 6145 & 6147 \\
\hline
\end{tabular}

(contingência em máquinas) podem ser tratadas de forma indireta via processamento pontual para cada espaço de estado cuja análise seja de interesse. A análise apresentada neste trabalho está focada apenas em contingências de linhas de transmissão para facilitar a compreensão dos efeitos da distribuição de probabilidades e do religamento.

A metodologia não contempla ainda nenhuma avaliação de custos. Entretanto, é possível inferir que o custo da melhoria da segurança do sistema está diretamente relacionado ao custo do redespacho de geração. No sistema teste utilizado não há nenhuma informação do custo de geração das usinas. Entretanto, transportando essa questão para a realidade brasileira, o redespacho poderia representar um significativo aumento do custo de geração na eventual elevação do despacho de unidades termoelétricas. Por outro lado, o aumento da segurança operativa representa um benefício considerável, uma vez que a eventual ocorrência de um corte de carga ou mesmo de um blecaute representa custos muito elevados para a sociedade.

\section{CONCLUSÕES}

A metodologia sugerida apresenta ganhos quando comparada às metodologias clássicas determinísticas encontradas na literatura, pois permite uma análise mais acurada do comportamento dinâmico do sistema elétrico. Além disso, ela possibilita também uma avaliação mais consistente do risco operativo do sistema por conta da análise estocástica das contingências.

A formulação de um problema de otimização tendo como função aptidão um índice estocástico da segurança dinâmica é inédita e só pôde ser desenvolvida a partir da criação do índice de estabilidade aparente (IEA), o qual consegue representar o desempenho dinâmico de um sistema elétrico de potência considerando diversos tipos de incertezas.

A principal vantagem dessa abordagem é a flexibilidade proporcionada pela utilização de metaheurísticas. O algoritmo enxame de partículas, em especial, requer a definição de poucos parâmetros, simplificando a sua implementação e utilização.

\section{AGRADECIMENTOS}

Os autores agradecem o apoio parcial do CNPq e da FAPERJ para a realização desse trabalho de pesquisa.

\section{APÊNDICE A}

\section{ÍNDICE DE ESTABILIDADE APARENTE (IEA)}

Antes de formular-se o indicador probabilístico denominado como Índice de Estabilidade Aparente (IEA), utilizado neste artigo, faz-se necessário apresentar alguns comentários sobre o conceito de margem de estabilidade de sistemas de potência. Maiores detalhes sobre os aspectos aqui apresentados podem ser obtidos em (Pavella et al., 2000; Groetaers dos Santos, 2009). Em geral, as margens de estabilidade de um sistema de potência expressam a que distância o sistema encontra-se da situação de estabilidade/instabilidade. Essa grandeza é obtenível pelo denominado método da máquina equivalente (SIME - "single machine equivalent") e resulta da generalização do conceito de "máquina contra barra infinita" (OMIB - "one machine infinite bus") e da aplicação do critério das áreas iguais. O método SIME, aqui utilizado para obter o IEA, baseia-se em um OMIB generalizado, tratando-se, portanto, de um método híbrido, temporaldireto. O sistema multimáquina é avaliado em sua evolução durante o transitório, com todo o detalhe inerente a simulação no tempo, enquanto infere-se sobre sua condição de estabilidade através do método das áreas iguais. $\mathrm{O}$ ponto crucial dessa abordagem reside na correta identificação do padrão de decomposição das máquinas do sistema em dois grupos, de forma a se definir o OMIB crítico. OMIBs generalizados são obtidos quando, em adição ao relaxamento da premissa de coerência, adota-se uma modelagem detalhada do sistema de potência. O modelo dinâmico é descrito pela equação (Apêndice A.1), porém a curva $P_{a} \times \delta$ não é mais senoidal. Não obstante, o conceito de energia do critério das áreas iguais permanece válido. 


$$
M \frac{d^{2} \delta}{d t^{2}}=P_{m}-P_{e}=P_{a}
$$

(Apêndice A.1)

onde $M$ é o coeficiente de inércia, $\delta$ o ângulo do rotor, $P_{m}$ a potência mecânica, $P_{e}$ a potência elétrica, $P_{a}$ a potência acelerante. $\mathrm{O}$ critério das áreas iguais fundamenta-se no conceito de energia. Basicamente, esse critério estabelece que as propriedades de estabilidade de um cenário de contingência podem ser obtidas em termos de uma margem de estabilidade, definida pela equação (Apêndice A.2) como o valor excedente de área desacelerante $\left(A_{d e s}\right)$ em relação à área acelerante $\left(A_{a c e}\right)$, no plano $P \times \delta$ da trajetória do OMIB. Assim,

$$
\eta=A_{d e s}-A_{a c e}
$$

(Apêndice A.2)

$\mathrm{A}$ área acelerante $\left(A_{a c e}\right)$ representa a energia cinética armazenada durante o período de defeito, enquanto que a área desacelerante $\left(A_{d e s}\right)$ representa a energia potencial máxima que o sistema pode dissipar durante o período pós- defeito. A diferença principal, e essencial, em relação ao critério das áreas iguais aplicado em sua forma clássica é que, para o método SIME, as curvas $P_{m} \times \delta$ e $P_{e} \times \delta$ do OMIB generalizado são computadas dos dados resultantes de uma simulação no tempo. Essa simulação é, em geral, realizada somente durante o curto espaço de tempo necessário para os cálculos de aplicação do critério das áreas iguais e caracterização da condição de estabilidade do sistema. Um OMIB de trajetória instável atinge um ângulo instável $\delta_{u}$, no tempo $t_{u}$, como indicado pela equação (Apêndice A.3):

$$
\left\{\begin{array}{l}
P_{a}\left(t_{u}\right)=0 \\
\dot{P}_{a}\left(t_{u}\right)=\frac{d P_{a}\left(t_{u}\right)}{d t} \mid>0
\end{array}\right.
$$

com $\omega>0$ (velocidade angular do rotor) para $t>t_{0}$. A equação (Apêndice A.3) determina uma condição de término antecipado da simulação no tempo. A trajetória de um OMIB estável atinge um ângulo de retorno $\delta_{r}\left(\delta_{r}<\delta_{u}\right)$, em $t=t_{r}$, conforme a equação (Apêndice A.4):

$$
w\left(t_{r}\right)=0, \operatorname{com} P_{a}\left(t_{r}\right)<0
$$

(Apêndice A.4)

A equação (Apêndice A.4) determina a condição de término antecipado da simulação no tempo por detecção de sistema estável. Finalmente, por definição, um OMIB com trajetória criticamente estável atinge o ângulo de retorno $\delta_{r}$ com
$P_{a}\left(t_{r}\right)=0$. A manipulação conveniente da equação (Apêndice A.5) conduz à margem instável $\eta_{u}$ na forma da equação (Apêndice A.6):

$$
\begin{array}{rlr}
M \frac{d^{2} \delta}{d t^{2}} & =P_{a}=P_{m}-P_{e}=P-P_{\max } & \sin (\delta-v) \\
\eta_{u} & =-\frac{1}{2} M w^{2} & \text { (Apêndice } A .5)
\end{array}
$$

A equação (Apêndice A.6) é muito simples e fácil de ser computada, requerendo apenas o conhecimento do coeficiente de inércia do OMIB e da velocidade do rotor. Condições extremamente severas podem conduzir a trajetórias pósdefeito que apresentem somente potência acelerante positiva. Nesses casos, outro tipo de margem deve ser utilizado para contornar essa dificuldade. De acordo com o comportamento do sistema as margens instáveis acima descritas são notadas, respectivamente, como margens de Tipo 1 ou Tipo 2. Ainda pela manipulação da equação (Apêndice A.5), observando que o OMIB permanece estável se $P_{e P}$ retorna antes de cruzar com $P_{m}$ e que $\omega=0$ para $\delta=\delta_{0}$ e $\delta=\delta_{r}$, a margem estável fica dada pela equação (Apêndice A.7):

$$
\eta_{e}=-\int_{\delta_{r}}^{\delta_{u}} P_{a} d \delta=\int_{\delta_{r}}^{\delta_{u}}\left|P_{a}\right| d \delta \quad \text { (Apêndice A.7) }
$$

A margem estável dada pela equação (Apêndice A.7) não pode ser calculada de forma exata dado que nem $\delta_{u}$ nem $P_{e P}(\delta)\left(\delta_{u}>\delta>\delta_{r}\right)$ podem ser computados diretamente, já que a curva $P_{e P} \times \delta$ do OMIB retorna em $\delta=\delta_{r}$. Duas formas possíveis de cálculo aproximado de $\eta_{e}$ envolvem, ou uma aproximação triangular da área desacelerante, ou a extrapolação de $P_{a}(\delta)$ via aproximação por mínimos quadrados entre $\delta_{r}$ e $\delta_{u}$. Essas considerações levam a concluir que: (i) Margens negativas só podem ser definidas quando existir $A_{d e s}\left(\right.$ ou $\delta_{u}$ ). Isso só acontece quando a potência elétrica $\left(P_{e}\right)$ ultrapassa a potência mecânica $\left(P_{m}\right)$ em algum instante $t \geq t_{e}$. A margem negativa Tipo 1 é expressa em MW.rd e a margem negativa Tipo 2 é dada em MW.; (ii) Margens positivas só podem ser definidas em casos próximos ao limite de estabilidade. Tendo como referência as condições que definem o limite de estabilidade para as quais $\eta=0$, nota-se que na acepção do método SIME, as margens de estabilidade variam entre pequenos valores positivos de $\eta$ e valores negativos limitados a $\left|\eta_{m x}\right|$ que corresponde a $P_{a m i n}=0$. Em situações muito estáveis não se pode caracterizar um OMIB (ou, equivalentemente, $\delta_{u}$ ). Por outro lado, em situações extremamente severas, a margem dada pelo critério das áreas iguais não mais existe em função de uma das seguintes condições: (i) As curvas de potência mecânica $\left(P_{m}\right)$ e de potência 
elétrica $\left(P_{e}\right)$ não se interceptam; (ii) $\mathrm{O}$ ângulo $\delta_{e}$ fica além do ângulo de instabilidade $\delta_{u}$. Para efeito dos cálculos computacionais, quando uma dessas condições se estabelece, um valor substituto para a margem é adotado, correspondendo ao valor mínimo de $P_{a}$ durante o transitório. Esse é um valor dado em unidades de potência (MW) e define a chamada margem do Tipo 2. O efeito das incertezas é introduzido pela obtenção da expectância de margem negativa, dada pela equação (Apêndice A.8), (Groetaers dos Santos, 2009):

$$
\bar{E}\left(M^{-}\right)=\sum_{i \in l} m_{i} p_{i}
$$

(Apêndice A.8)

onde, $\bar{E}\left(M^{-}\right)$é a expectância de margem negativa; $m_{i}$ é a margem negativa de estabilidade do i-ésimo estado instável; $p_{i}$ é a probabilidade do i-ésimo estado instável; ( é o conjunto de estados instáveis contabilizados do espaço probabilístico (estados avaliados com margens negativas). A expectância de margem negativa deve ser contabilizada separadamente para as margens de Tipo 1 e de Tipo 2, resultando em expectâncias de margens negativas de Tipo 1 e de Tipo $2\left(\bar{E}\left(M_{1}^{-}\right)\right.$ e $\bar{E}\left(M_{2}^{-}\right)$). A $E M N$ do Tipo 1 é expressa em MW.rd e a $E M N$ do Tipo 2 é expressa em MW. Ambas representam o grau de insegurança dinâmica do sistema. As expectâncias de margem de estabilidade (Tipo 1 e Tipo 2) isoladamente podem ser de difícil interpretação para produção de um diagnóstico do grau de insegurança dinâmica do sistema por serem grandezas obtidas em unidades distintas e também por carregarem informações diferentes sobre a natureza do comportamento dinâmico do sistema. Porém, representando-se o módulo da expectância de margem negativa do Tipo 1 segundo um eixo horizontal e o módulo da expectância de margem negativa de Tipo 2 segundo um eixo vertical, esses módulos podem ser compostos conforme a equação (Apêndice A.9), definindo assim o IEA. O sinal negativo tem o objetivo de situar a resultante no terceiro quadrante, enfatizando que o indicador advém da composição de grandezas essencialmente negativas.

$$
I E A=\sqrt{\bar{E}\left(M_{1}^{-}\right)^{2}+\bar{E}\left(M_{2}^{-}\right)^{2}}
$$

(Apêndice A.9)

O IEA é portanto um número menor ou igual a zero que indica o grau de segurança do sistema. Quanto mais próximo de zero, maior o grau de segurança do sistema. O índice será numericamente igual a $\bar{E}\left(M_{1}^{-}\right)$quando o sistema só apresentar margens negativas do Tipo $1 \mathrm{ou}$, por outro lado, igual a $\bar{E}\left(M_{2}^{-}\right)$quando o sistema só apresentar margens negativas do Tipo 2. O índice IEA é capaz de reproduzir o efeito das variações isoladas ou conjuntas das margens de Tipo 1 e de Tipo 2 sobre o risco, no sentido da diminuição ou do aumento da segurança do sistema. Um conjunto abrangente de aplicações exitosas deste indicador podem ser vistas em (Groetaers dos Santos, 2009).

\section{REFERÊNCIAS}

Abido, A. (2001). Particle swarm optimization for multimachine power system stabilizer design, Power Engineering Society Summer Meeting, 2001, Vol. 3, Vancouver, BC, pp. 1346-1351.

Anderson, P. M., Bose, A., Timo, K. J. and Villaseca, F. E. (1983). Probabilistic approach to stability analysis. volume 1: Mathematical models, computing methods, and results, OSTI ID: 6438311; Legacy ID: DE83901466 EPRI-EL-2797-Vol.1, Arizona State Univ., Tempe (USA). Dept. of Electrical and Computer Engineering.

Assis, T., Falcao, D. and Taranto, G. (2007). Dynamic transmission capability calculation using integrated analysis tools and intelligent systems, IEEE Transactions on Power Systems 22(4): 1760-1770.

Athay, T., Podmore, R. and Virmani, S. (1979). A practical method for the direct analysis of transient stability, IEEE Transactions on Power Apparatus and Systems PAS-98(2): 573-584.

Chen, M. J., Wu, B. and Chen, C. (2009). Determination of shortest distance to voltage instability with particle swarm optimization algorithm, European Transactions on Electrical Power 19(8): 1109-1117.

Feo, T. A. and Resende, M. G. C. (1995). Greedy randomized adaptive search procedures, Journal of Global Optimization 6(2): 109-133.

Glover, F. (1989). Tabu search - part i, ORSA Journal on Computing 1(3): 190-206.

Glover, F. (1990). Tabu search - part ii, ORSA Journal on Computing 2(1): 4-32.

Groetaers dos Santos, M. (2009). Segurança de Sistemas de Potência em Regime Transitório Considerando Incertezas, $\mathrm{PhD}$ thesis, IC/UFF.
URL: http://www.ic.uff.br/ PosGraduacao/lista_teses.php?ano= 2009

Jardim, J. L. A. (1994). Advances in Power System Transient Stability Assessment Using Transient Energy Function Methods., PhD thesis, Imperial College, London. 
Ju, P., Handschin, E. and Karlsson, D. (1996). Nonlinear dynamic load modelling: model and parameter estimation, IEEE Transactions on Power Systems 11(4): 1689-1697.

Kamwa, I., Grondin, R. and Loud, L. (2001). Time-varying contingency screening for dynamic security assessment using intelligent-systems techniques, IEEE Transactions on Power Systems 16(3): 526-536.

Karapidakis, E. and Hatziargyriou, N. (2000). Application of artificial neural networks for security assessment of medium size power systems, 10th Mediterranean Electrotechnical Conference, 2000. MELECON 2000., Vol. 3, pp. 1189-1192.

Kennedy, J. and Eberhart, R. (1995). Particle swarm optimization, IEEE International Conference on Neural Networks, 1995. Proceedings., Vol. 4, pp. 1942-1948.

Kundur, P. (1994). Power System Stability and Control, McGraw-Hill Inc.

Kuo, D.-H. and Bose, A. (1995). A generation rescheduling method to increase the dynamic security of power systems, IEEE Transactions on Power Systems 10(1): 6876.

Lee, K. Y. and El-Sharkawi, M. A. (2008). Modern Heuristic Optimization Techniques Theory and Applications to Power Systems, John Willey, IEEE Press, New Jersey.

Mohammadi, S., Gharaveisi, A., Mashinchi, M. and Rafiei, S. (2009). New evolutionary methods for optimal design of pid controllers for avr system, IEEE Bucharest PowerTech, 2009., pp. 1-8.

ONS (2009). Procedimentos de rede - submódulo 23.3 diretrizes e critérios para estudos elétricos. Operador Nacional do Sistema Elétrico.

Pai, M. (1989). Energy Function Analysis for Power System Stability, Kluwer Academic Publishers.

Panigrahi, B., Abraham, A. and Das, S. (2010). Computational Intelligence in Power Engineering, Vol. 302 of Studies in Computational Intelligence, 1 edn, SpringerVerlag.

Pavella, M., Ernst, D. and Ruiz-Vega, D. (2000). Transient Stability Of Power Systems - A Unified Approach To Assessment And Control, Kluwer Academic Publishers.

Rudolf, A. and Bayrleithner, R. (1999). A genetic algorithm for solving the unit commitment problem of a hydrothermal power system, IEEE Transactions on Power Systems 14(4): 1460-1468.
Sant'anna, E., Souza, A., Takahata, A., Assis, T., Schilling, M. and Passos Filho, J. (2008). Limites de intercâmbio em sistemas de grande porte via otimização, XVII Congresso Brasileiro de Automática (CBA 2008), Juiz de Fora, MG, pp. 1-6.

Sarimveis, H. and Nikolakopoulos, A. (2005). A line up evolutionary algorithm for solving nonlinear constrained optimization problems, Computers \& Operations Research 32(6): 1499-1514.

Schilling, M., Billinton, R. and dos Santos, M. (2009). Bibliography on power systems probabilistic security analysis 1968-2008, International Journal of Emerging Electric Power Systems 10(3): 1-48.

Silva Neto, A. and Becceneri, J. (2009). Técnicas de Inteligência Computacional Inspiradas na Natureza - Aplicação em Problemas Inversos em Transferência Radiativa, Vol. 41 of Notas em Matemática Aplicada, São Carlos, SP, Brasil.

Sun, Y., Xinlin, Y. and Wang, H. (2004). Approach for optimal power flow with transient stability constraints, IEE Proceedings Generation, Transmission and Distribution 151(1): 8-18.

Takahata, A. (2008). Segurança de Sistemas de Potência sob Pequenas Perturbações Considerando Incertezas, $\mathrm{PhD}$ thesis, COPPE/UFRJ.

URL: http://www.pee.ufrj.br/teses/ index.php

Valette, A., Huang, J., Guillon, S., Loud, L., Vanier, G., Levesque, F., Riverin, L., Rizzi, J.-C. and Guillemette, F. (2008). An integrated approach for optimizing dynamic transfer limits at hydro-quebec, IEEE Power and Energy Society General Meeting - Conversion and Delivery of Electrical Energy in the 21st Century, 2008., pp. 1-8.

Voumvoulakis, E. and Hatziargyriou, N. (2010). A particle swarm optimization method for power system dynamic security control, IEEE Transactions on Power Systems 25(2): 1032-1041.

Wang, D. and Yu, Y. (2008). Dynamic security risk assessment and optimization of power transmission system, Proceedings of the 10th International Conference on Probabilistic Methods Applied to Power Systems, 2008. PMAPS '08., pp. 1-6.

Zarate-Minano, R., Van Cutsem, T., Milano, F. and Conejo, A. (2010). Securing transient stability using time-domain simulations within an optimal power flow, IEEE Transactions on Power Systems 25(1): 243 -253. 